\title{
Note on the Eilenberg-Moore Spectral Sequence
}

\author{
By
}

\author{
Atsushi YAMAGUCHI*
}

\begin{abstract}
In this paper, we prove two theorems on the Eilenberg-Moore spectral sequence. We give a relation between the Bockstein homomorphism in the $E^{2}$-term and the Bockstein homomorphism of the space to which the spectral sequence converges and also relate the homology suspension to the Eilenberg-Moore spectral sequence.
\end{abstract}

\section{Introduction}

The purpose of this note is to prove two theorems on the Eilenberg-Moore spectral sequence; one relates the algebraic Bockstein homomorphism between the $E^{2}$-terms to the geometric Bockstein homomorphism between the homologies of the spaces to which the spectral sequences converge (Theorem 2.2), and the other relates the homology suspension to the Eilenberg-Moore spectral sequence associated with a path fibration (Theorem 3.5).

In order to prove these theorems, we recall the definition of the EilenbergMoore spectral sequence. After the original work of Eilenberg and Moore ([2]), various constructions have been done by Hodgkin ([4]), Smith ([7], [8]), Rector ([6]) and Heller ([3]). In this note, we adopt a point of view of Hodgkin and Smith who construct the Eilenberg-Moore spectral sequence as the Künneth spectral sequence on the category of pointed spaces over some fixed base space.

In Section 1, we construct the Künneth spectral sequence for a generalized homology theory, dualizing the argument of Smith ([8]). We give a sufficient condition for convergence of the spectral sequence ((1.11), (1.23) (ii)) and one for identification of the $E^{2}$-term ((1.17), (1.22)). These results are also obtained in [3] under a categorical framework. Our concrete construction enables us to prove the main theorems in the following sections.

Communicated by N. Shimada, March 14, 1986.

* Department of Mathematics, University of Osaka Prefecture, Sakai 591, Japan. 
In Section 2, we prove that the algebraic Bockstein homomorphism between the $E^{2}$-terms of the Künneth spectral sequence "converges" to the geometric Bockstein homomorphism. Our assertion (2.2) is quite similar to the main theorem on the boundary homomorphism of [11], although the proof is much easier.

In Section 3, we consider the Eilenberg-Moore spectral sequence for a generalized homology $h_{*}$, associated with the path fibration over a space $B$. Then, under suitable assumptions, there is a homomorphism $\tilde{h}_{n}(\Omega B) \rightarrow E_{-1, n+1}^{2}$ and a natural equivalence $E_{-1, n+1}^{2} \rightarrow P h_{n+1}(B)$. We show that the composition of these coincides with the homology suspension.

The results of Sections 2, 3 are applied to determine the structure of the homology of double loop spaces of complex Stiefel manifolds ([10]).

I would like to thank J. P. Mayer, J. M. Boardman, J. Morava and W. S. Wilson for useful conversations and encouragement. And I am also grateful to A. Kono for helpful suggestions and his interest on the materials of Sections 2 and 3.

\section{$\S 1$. Recollections on the Eilenberg-Moore Spectral Sequence}

First, we define a category $\operatorname{Top}_{*} / B$ to formulate the Eilenberg-Moore spectral sequence as the Künneth spectral sequence in this category. We will use the notations and some results of Section 1 of [8]. (See also [4], [7].)

Definition 1.1 ([8]). Let $B$ be a fixed topological space. We define a category of pointed spaces over $B$, denoted by $\mathbb{T o p}_{*} / B$ as follows. An object of Top $_{*} / B$ is a pair of maps $(f, s)(f: T(f) \rightarrow B, s: B \rightarrow T(f))$ between topological spaces $T(f)$ and $B$ such that $f_{\circ} s=1_{B}$ and $s B$ is a neighborhood deformation retract of $T(f)$. A morphism $\varphi:(f, s) \rightarrow(g, t)$ of Top* $/ B$ consists of a continuous map $T(\varphi): T(f) \rightarrow T(g)$ such that $g \circ T(\varphi)=f, T(\varphi) \circ s=t$.

We denote by Top $_{*}$ the category of pointed topological spaces with nondegrerate basepoints. We define functors $\Gamma: \operatorname{Top}_{*} \rightarrow \operatorname{Top}_{*} / B$ and $\Phi: \operatorname{Top}_{*} / B$ $\rightarrow$ Top $_{*}$ which play a central role in the construction of the Eilenberg-Moore spectral sequence.

Definition 1.2 ([8]). For each pointed topological space $\left(X, x_{0}\right)$, let $p: B \times X \rightarrow B$ be the projection onto $B$ and let $s: B \rightarrow B \times X$ be the canonical inclusion $s(b)=\left(b, x_{0}\right)$. We define a functor $\Gamma$ by $\Gamma\left(X, x_{0}\right)=(p, s)$. And we 
define a functor $\Phi$ by $\Phi(f, s)=(T(f) / s B, s B / s B)$.

Lemma 1.3. The functor $\Gamma$ is a right adjoint of $\Phi$; that is, there is a natural equivalence

$$
\operatorname{Mor}_{\mathbb{T o p}^{*} / B}\left((f, s), \Gamma\left(X, x_{0}\right)\right) \cong \operatorname{Mor}_{\mathbb{T o p} *}\left(\Phi(f, s),\left(X, x_{0}\right)\right) .
$$

Proof. We construct a natural map $\alpha: \operatorname{Mor}_{\mathbb{T}^{*} / B}\left((f, s), \Gamma\left(X, x_{0}\right)\right)$ $\rightarrow \operatorname{Mor}_{\text {Top* }}\left(\Phi(f, s),\left(X, x_{0}\right)\right)$ as follows. Let $\varphi:(f, s) \rightarrow \Gamma\left(X, x_{0}\right)$ be a morphism of $\operatorname{Top}_{*} / B$, and let $q: B \times X \rightarrow X$ be the projection onto $X$. Then the composition $T(f) \stackrel{T(\varphi)}{\longrightarrow} B \times X \stackrel{q}{\rightarrow} X$ maps $s B$ to the base point $x_{0}$. We define $\alpha(\varphi)$ to be the map $T(f) / s B \rightarrow X$ induced by $q \circ T(\varphi)$. The inverse $\alpha^{-1}$ of $\alpha$ is described as follows. For a morphism $\psi: \Phi(f, s) \rightarrow\left(X, x_{0}\right)$, we put $T\left(\alpha^{-1}(\psi)\right)(x)=(f(x)$, $\psi \circ \pi(x))$ where $\pi: T(f) \rightarrow T(f) / s B$ is the canonical projection.

In the category $\mathbb{T o p}_{*} / B$, we can construct mapping cones, suspensions, products, smash products and other constructions which we usually do in the category $\mathbb{T O P}_{*}$. And we can define a cofiber sequence in $\mathbb{T} \mathscr{O P} * / B$ as in $\mathbb{T} \mathscr{Q P} *$. Here we give the constructions of mapping cones, products, smash products, and suspensions. (See Section 1 of [8] for details.)

Constructions $\mathbb{1}_{\circ} \mathbb{4}_{\text {。 }}$ (i) Let $\varphi:(f, s) \rightarrow(g, t)$ be a morphism of $\mathbb{T} \circ \mathbb{p}_{*} / B$. We define the mapping cone of $\varphi$, denoted by $\left(C_{B}(\varphi), S_{C_{B}(\varphi)}\right)$ as follows:

$$
T C_{B}(\varphi)=(T(f) \times I) \Perp T(g) \mid \begin{aligned}
& (x, 1) \sim T \varphi(x): x \in T(f) \\
& (s(b), r) \sim t(b): b \in B, r \in \mathbb{I} \\
& \left(x^{\prime}, 0\right) \sim\left(x^{\prime \prime}, 0\right) \text { if } f\left(x^{\prime}\right)=f\left(x^{\prime \prime}\right) \\
& \text { for } x^{\prime}, x^{\prime \prime} \in \mathbb{T}(f)
\end{aligned}
$$

$C_{B}(\varphi): T C_{B}(\varphi) \rightarrow B$ and $S_{C_{B}(\varphi)}: B \rightarrow T C_{B}(\varphi)$ are defined by $C_{B}(\varphi)([x, r])=f(x)$ for $x \in \mathbb{T}(f), r \in \mathbb{I}, C_{B}(\varphi)([y])=g(y)$ for $y \in \mathbb{T}(g)$ and $S_{C_{B}(\varphi)}(b)=[t(b)]$ for $b \in B$ where $[x, r]$ and $[y]$ are the elements of $\operatorname{TC}_{B}(\varphi)$ represented by $(x, r) \in \mathbb{T}(f) \times \mathbb{I}$ and $y \in T(g)$ respectively. Note that there is a natural inclusion $\iota:(g, t) \rightarrow$ $\left(C_{B}(\varphi), S_{C_{B}(\varphi)}\right)$ defined by $T(\iota)(y)=[y]$.

(ii) Let $(f, s)$ and $(g, t)$ be pointed space over $B$, their product $(f, s) \stackrel{\pi_{f}}{\longleftarrow}$ $(f, s) \underset{B}{\times}(g, t) \underset{\pi_{g}}{\longrightarrow}(g, t)$ is defined by the following; We put $(f, s) \underset{B}{\times}(g, t)=(f \underset{B}{\times} g$, $\left.s_{B} \times t\right) \quad$ and $\quad T(f \underset{B}{\times} g)=T(f) \times \underset{B}{*} T(g)=\{(x, y) \in T(f) \times T(g) \mid f(x)=g(y)\} . \quad f \times{ }_{B} g:$

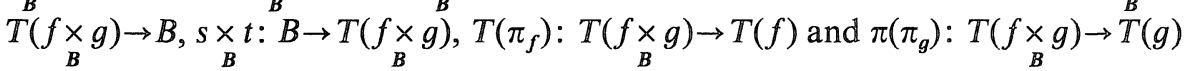
are given by $f_{B} \times g(x, y)=f(x)=g(y), s_{B} t(b)=(s(b), t(b)), T\left(\pi_{f}\right)(x, y)=x$ and $T\left(\pi_{g}\right)(x, y)=y$. 
(iii) Let $(f, s)$ and $(g, t)$ as above. We define their smash product $(f, s) \underset{B}{\wedge}$ $(g, t)=(\underset{B}{\wedge} g, s \wedge t)$ by $T(f \underset{B}{\wedge} g)=T\left(\underset{B}{\times_{B}} g\right) /(x, t(b)) \sim(s(b), y)$ for $(x, y) \in T(f \underset{B}{\wedge} g), b \in B . \quad f \underset{B}{\wedge} g: T(f \underset{B}{\wedge} g)$ $\rightarrow B$ and $s \wedge t: B \rightarrow T\left(f \wedge_{B} g\right)$ are given by $f \wedge_{B} g\left(x \wedge_{B} y\right)=f(x)=g(y)$ and $s \wedge_{B} t(b)$ $=s(b) \wedge t(b)$.

(iv) Let $(f, s)$ be a pointed space over $B$. Let us define a suspension functor $\operatorname{Top}_{*} / B \rightarrow \operatorname{Top}_{*} / B$ by $\sum_{B}(f, s)=\Gamma\left(S^{1}, *\right) \underset{B}{\wedge}(f, s)$, where $\left(S^{1}, *\right)$ is a circle with a base point $*$.

We give some propositions we need without proofs.

Propositions 1.5 ([8]). (i) The functor $\Phi:$ Top $_{*} / B \rightarrow$ Top $_{*}$ preserves cofibrations.

(ii) Let $\underset{B}{\wedge}: \operatorname{Top}_{*} / B \times \operatorname{Top}_{*} / B \rightarrow \operatorname{Top}_{*} / B$ be the smash product over $B$ For any pointed space $(f, s)$ over $B$, the functors $(f, s) \underset{B}{\wedge}(-),(-) \wedge_{B}(f, s)$ : $\operatorname{Top}_{*} / B \rightarrow$ Top $_{*} / B$ preserve cofibrations.

(iii) Let $\sum=\sum_{B}$ : $\operatorname{Top}_{*} / B \rightarrow \mathbb{T o p}_{*} / B$ be the suspension functor on $\mathbb{T o p}_{*} / B$, then there is a natural map $\Delta:\left(h^{\prime \prime}, u^{\prime \prime}\right) \rightarrow \sum\left(h^{\prime}, u^{\prime}\right)$ for each cofiber sequence $\left(h^{\prime}, u^{\prime}\right) \stackrel{i}{\longrightarrow}(h, u) \stackrel{i}{\longrightarrow}\left(h^{\prime \prime}, u^{\prime \prime}\right)$ in $\operatorname{Top}_{*} / B$ such that $(h, u) \stackrel{j}{\longrightarrow}\left(h^{\prime \prime}, u^{\prime \prime}\right) \stackrel{\Delta}{\longrightarrow} \sum\left(h^{\prime}, u^{\prime}\right)$ and $\left(h^{\prime \prime}, u^{\prime \prime}\right) \stackrel{\Delta}{\longrightarrow} \sum\left(h^{\prime}, u^{\prime}\right) \stackrel{\Sigma i}{\longrightarrow} \sum(h, u)$ are cofiber sequences.

(iv) $\sum_{B}$ preserves cofibrations and commutes with $\Phi$ : that is, $\Phi \circ \sum_{B}$ $=\sum \circ \Phi$, where $\sum$ in the right hand is the usual suspension functor on $\operatorname{Top}_{*}$.

Definition 1.6. Consider the natural transformation $\varphi=\alpha^{-1}\left(1_{\Phi}\right): 1_{\text {Top } * / B}$ $\rightarrow \Gamma \circ \Phi$. For a pointed space $(f, s)$ over $B$, let $C_{\varphi}(f, s)$ be the mapping cone of $\varphi_{(f, s)}:(f, s) \rightarrow \Gamma \circ \Phi(f, s)$, and let $\iota_{(f, s)}: \Gamma \circ \Phi(f, s) \rightarrow C_{\varphi}(f, s)$ be the natural inclusion. Thus we define a functor $C_{\varphi}: \mathbb{T o p}_{*} / B \rightarrow \mathbb{T o p}_{*} / B$ and a natural cofiber sequence $(f, s) \stackrel{\varphi(f, s)}{\longrightarrow} \Gamma \circ \Phi(f, s) \stackrel{\iota(f, s)}{\longrightarrow} C_{\varphi}(f, s)$.

Let $\tilde{h}_{*}$ be a reduced homology theory on $\operatorname{Top}_{*}$. Putting ${ }_{B} h_{*}=\tilde{h}_{*} \circ \Phi$, we have a homology theory ${ }_{B} h_{*}$ on $\operatorname{Top}_{*} / B([8]$, Corollary 2.2).

Construction 1.7. Let $\tilde{h}_{*}$ and ${ }_{B} h_{*}$ as above, and let $(f, s)$ and $(g, t)$ be pointed spaces over $B$. We form a sequence of natural cofibrations.

$$
\begin{aligned}
& (f, s) \stackrel{\varphi}{\longrightarrow} \Gamma \circ \Phi(f, s) \stackrel{\iota}{\longrightarrow} C_{\varphi}(f, s) \\
& C_{\varphi}(f, s) \stackrel{\varphi}{\longrightarrow} \Gamma \circ \Phi C_{\varphi}(f, s) \stackrel{\iota}{\longrightarrow} C_{\varphi}^{2}(f, s), \text { where } C_{\varphi}^{i}=\overbrace{C_{\varphi}^{\circ \cdots \circ C_{\varphi}}}^{i \text { fact ors }} \\
& \quad(i=0,1,2, \ldots) \\
& C_{\varphi}^{i}(f, s) \stackrel{\varphi}{\longrightarrow} \Gamma \circ \Phi C_{\varphi}^{i}(f, s) \stackrel{\iota}{\longrightarrow} C_{\varphi}^{i+1}(f, s)
\end{aligned}
$$


We apply the functor $(g, t) \wedge(-)$ to the above cofibrations to have the following sequence of cofibrations by (1.5).

$$
\begin{aligned}
& (g, t) \underset{B}{\wedge}(f, s) \underset{B}{\stackrel{1 \wedge \varphi}{\longrightarrow}}(g, t) \underset{B}{\wedge} \Gamma \circ \Phi(f, s) \stackrel{1 \wedge \iota}{\longrightarrow}(g, t) \wedge C_{\varphi}(g, t) \\
& (g, t){ }_{B}^{\wedge} C_{\varphi}(f, s) \stackrel{1 \wedge \varphi}{\longrightarrow}(g, t) \underset{B}{\wedge} \Gamma \circ \Phi C_{\varphi}(f, s) \stackrel{1 \wedge \iota}{\longrightarrow}(g, t) \underset{B}{\wedge} C_{\varphi}^{2}(g, t) \\
& (g, t) \wedge_{B}^{\wedge} C_{\varphi}^{i}(f, s) \stackrel{1 \wedge \varphi}{\longrightarrow}(g, t){\underset{B}{\wedge}}_{\Gamma} \Gamma \Phi C_{\varphi}^{i}(f, s) \stackrel{1 \wedge \iota}{\longrightarrow}(g, t){\underset{B}{\wedge}}_{\varphi} C_{\varphi}^{i+1}(g, t)
\end{aligned}
$$

Thus we obtain the following long exact sequence for each $i=0,1,2, \ldots$.

$$
\begin{aligned}
& \longrightarrow{ }_{B} h_{q+1}\left((g, t) \wedge \wedge_{B} C_{\varphi}^{i+1}(f, s)\right) \stackrel{\Delta \bullet}{\longrightarrow}{ }_{B} h_{q}\left((g, t) \wedge_{B} C_{\varphi}^{i}(f, s)\right) \stackrel{(1 \wedge \varphi)_{*}}{\longrightarrow} \\
& { }_{B} h_{q}\left((g, t) \underset{B}{\wedge} \Gamma \circ \Phi C_{\varphi}^{i}(f, s)\right) \stackrel{(1 \wedge \iota)_{+}}{\longrightarrow}{ }_{B} h_{q}\left((g, t){ }_{B} C_{\varphi}^{i+1}(f, s)\right) \stackrel{\Delta \bullet}{\longrightarrow} \cdots
\end{aligned}
$$

We set $D_{p, q}^{1}={ }_{B} h_{q}\left((g, t) \underset{B}{\wedge} C_{\varphi}^{-p}(f, s)\right), E_{p, q}^{1}={ }_{B} h_{q}\left((g, t) \underset{B}{\wedge} \Gamma \circ \Phi C_{\varphi}^{-p}(f, s)\right)(p=0,-1$, $-2, \ldots, q \in \mathbb{Z})$. The Künneth spectral sequence in the category $\mathbb{T O P}_{*} / B$ is defined to be the spectral sequence associated with the exact couple $\left\langle D_{p, q}^{1}\right.$, $\left.\mathbb{E}_{p, q}^{1},(1 \wedge \varphi)_{*},(1 \wedge \iota)_{*}, \Delta_{*}\right\rangle$.

To discuss the convergence problem, we have to define a filtration on ${ }_{B} h_{*}((g, t) \wedge(f, s))$. Considering the suspension category associated with the category $\mathbb{T}_{\mathbb{P}} / B([3])$, we "desuspend" the map $(g, t) \wedge C_{\varphi}^{i+1}(f, s) \stackrel{1 \wedge \Delta}{\longrightarrow}$ $(g, t) \wedge \sum C_{\dot{B}}^{i}(f, s) \cong \sum(g, t) \wedge_{B} C_{\varphi}^{i}(f, s)$ and obtain a map $\sum^{-1}(g, t) \wedge_{B} C_{\varphi}^{i+1}(f, s)$ $\rightarrow(g, t) \wedge C_{\varphi}^{i}(f, s)$. Thus we have the following sequence of maps of the suspension category.

$$
\begin{aligned}
& (g, t) \wedge \underset{B}{\wedge}(f, s) \longleftarrow \Sigma^{-1}(g, t) \wedge{ }_{B} C_{\varphi}(f, s) \longleftarrow \Sigma^{-2}(g, t) \wedge{ }_{B} C_{\varphi}^{2}(f, s) \longleftarrow \cdots \\
& \longleftarrow \Sigma^{-i}(g, t) \underset{B}{\wedge} C_{\varphi}^{i}(f, s) \longleftarrow \Sigma^{-i-1}(g, t) \underset{B}{\wedge} C_{\varphi}^{i+1}(f, s) \longleftarrow \cdots
\end{aligned}
$$

We put $F_{p, q}=\operatorname{Im}\left\{{ }_{B} h_{q}\left((g, t){ }_{B}^{\wedge} C_{\varphi}^{-p}(f, s)\right) \cong{ }_{B} h_{p+q}\left(\sum p(g, t) \underset{B}{\wedge} \mathbb{C}_{\varphi}^{-p}(f, s)\right) \longrightarrow\right.$ $\left.{ }_{B} h_{p+q}((g, t) \underset{B}{\wedge}(f, s))\right\}(p \leqq 0, q \in \mathbb{Z})$

$$
\begin{aligned}
& A_{p, q}=\operatorname{Im}\left\{{ }_{B} h_{q}\left((g, t) \underset{B}{\wedge} \Gamma \circ \Phi C_{\varphi}^{-p}(f, s)\right) \rightarrow{ }_{B} h_{q}\left((g, t) \underset{B}{\wedge} C_{\varphi}^{-p+1}(f, s)\right)\right\} \\
& \cap\left[\cap_{r \geqq 1} \operatorname{Im}\left\{{ }_{B} h_{q}\left(\sum^{-r}(g, t) \underset{B}{\wedge} C_{\varphi}^{-p+r+1}(f, s)\right) \rightarrow_{B} h_{q}\left((g, t) \underset{B}{\wedge} C_{\varphi}^{-p+1}(f, s)\right)\right\}\right] .
\end{aligned}
$$

By the construction of the spectral sequence, we have $E_{p, q}^{1-p} \supset E_{p, q}^{2-p} \supset \cdots \supset \mathbb{E}_{p, q}^{r} \supset$ $E_{q, p}^{r+1} \supset \cdots$. We set $E_{p, q}^{\infty}=\bigcap_{r \geqq 1-p} \mathbb{E}_{p, q}^{r}$, and note that ${ }_{B} h_{n}\left((g, t) \wedge_{B}(f, s)\right)=\mathbb{F}_{0, n}$ $\supset F_{-1, n+1} \supset \cdots \supset F_{m, n-m} \supset F_{m-1, n-m+1} \supset \cdots$.

Proposition 1.8. There is a short exact sequence 


$$
0 \longrightarrow F_{p, q} / F_{p-1, q+1} \longrightarrow E_{p, q}^{\infty} \longrightarrow A_{p, q} \longrightarrow 0 \quad(p \leqq 0, q \in \mathbb{Z}) .
$$

See [9] p. $464 \sim$ p. 470 for a proof.

Remark 1.9. There is an edge homomorphism ${ }_{B} h_{n}((g, t) \underset{B}{\wedge}(f, s))=F_{0, n}$ $\rightarrow F_{0, n} / F_{-1, n+1} \rightarrow E_{0, n}^{\infty} \subset E_{0, n}^{2}$.

Propositions 1.10. Let $(f, s),(g, t)$ be pointed spaces over $B$, then the following facts hold.

(i) $\Phi \circ \Gamma \circ \Phi(f, s)=\left(B_{+}\right) \wedge T(f) / s B$, where $B_{+}=B \Perp\{*\}$ (disjoint union). Hence ${ }_{B} h_{*}(\Gamma \circ \Phi(f, s))=\tilde{h}_{*}\left(\left(B_{+}\right) \wedge T(f) / s B\right)$.

(ii) Let $T(g) \underset{B}{\times} T(f)=\{(y, x) \in T(g) \times T(f) \mid g(y)=f(x)\}$, then $\Phi\left((g, t) \wedge_{B}\right.$ $(f, s))=T(g) \underset{B}{\times} T(f) / \bar{t} T(g) \cup \bar{s} T(f)$ where $\bar{t}: T(g) \rightarrow T(g) \underset{B}{\times} T(f), \bar{s}: T(f) \rightarrow T(g) \underset{B}{\stackrel{B}{B}}$ $T(f)$ are maps defined by $\bar{t}(y)=(y, \operatorname{sog}(y)), \bar{s}(x)=(t \circ f(x), x)$.

(iii) $\Phi((g, t) \underset{B}{\wedge} \Gamma \circ \Phi(f, s))$ is naturally homeomorphic to $T(g) / t B \wedge T(f) / s B$ $=T(g) \times T(f) / T(g) \times s B \cup t B \times T(f)$.

(iv) The map $(1 \wedge \varphi)_{*}:{ }_{B} h_{q}((g, t) \wedge(f, s)) \rightarrow{ }_{B} h_{q}\left((g, t) \wedge{ }_{B} \circ \Phi(f, s)\right)$ coincides with the map $\tilde{h}_{y}(T(g) \times \underset{B}{ } T(f) / \bar{t} T(g) \cup \bar{s} T(f)) \rightarrow \tilde{h}_{q}(T(g) \times T(f) / T(g) \times s B \cup t B \times$ $T(f))$ induced by the inclusion $T(g) \underset{B}{\times} T(f) \subset T(f) \times T(g)$.

Proofs are immediate from the definitions.

Lemma 1.11. Let $B$ be a simply connected space and let $(f, s)$ be a pointed space over $B$ such that ${ }_{B} H_{i}(f, s)=\widetilde{H}_{i}(T(f) / s B)=0$ for $i<k$. Then ${ }_{B} H_{i}\left(C_{\varphi}(f, s)\right)=0$ for $i<k+2$, where $\tilde{H}_{i}$ is the ordinary homology theory.

Proof. By the Künneth theorem of the ordinary homology, we have ${ }_{B} H_{\iota}(\Gamma \circ \Phi(f, s))=\widetilde{H}_{i}\left(\left(B_{+}\right) \wedge T(f) / s B\right)=0$ for $i<k$ and the smash product $\tilde{H}_{0}\left(B_{+}\right)$ $\otimes \tilde{H}_{i}(T(f) / s B) \rightarrow \tilde{H}_{i}\left(\left(B_{+}\right) \wedge T(f) / s B\right)$ is an isomorphism for $i=k, k+1$. It follows that $(\varepsilon \wedge 1)_{*}:{ }_{B} H_{i}(\Gamma \circ \Phi(f, s))=\widetilde{H}_{i}\left(\left(B_{+}\right) \wedge T(f) / s B\right) \rightarrow \widetilde{H}_{i}\left(S^{0} \wedge T(f) / s B\right)$ $={ }_{B} H_{i}(f, s)$ is an isomorphism for $i<k+2$, where $\varepsilon: B_{+} \rightarrow S^{0}$ is the collapsing map. Since the composition ${ }_{B} H_{i}(f, s) \stackrel{\varphi_{\star A}}{\longrightarrow} H_{i}(\Gamma \circ \Phi(f, s)) \stackrel{(\varepsilon \wedge 1)_{t}}{\longrightarrow} H_{B}(f, s)$ is the identity map, $\varphi_{*}$ is an isomorphism for $i<k+2$. Consider the long exact sequence associated with the cofibration $(f, s) \stackrel{\varphi}{\longrightarrow} \Gamma \circ \Phi(f, s) \stackrel{\iota}{\longrightarrow} C_{\varphi}(f, s)$, then the result follows.

Corollary 1.12. Let $\tilde{h}_{*}$ be a connective homology theory and let $B$ be a simply connected space. For any pointed space $(f, s)$ over $B,{ }_{B} \tilde{h}_{i}\left(C_{\varphi}^{p}(f, s)\right)=0$ for $i<2 p$.

Proof. Applying 1.11, we see that ${ }_{B} H_{i}\left(C_{\varphi}^{p}(f, s)\right)=0$ for $i<2 p$ by induction 
on $p$. Then we have $\tilde{H}_{i}\left(\Phi C_{\varphi}^{p}(f, s) ; \tilde{h}_{*}\left(S^{0}\right)\right)=0$ for $i<2 p$ by the universal coefficient theorem of the ordinary homology. Consider the Atiyah-Hirzebruch spectral sequence $\tilde{H}_{i}\left(\Phi C_{\varphi}^{p}(f, s) ; \tilde{h}_{j}\left(S^{0}\right)\right) \Rightarrow \tilde{h}_{i+j}\left(\Phi C_{\varphi}^{p}(f, s)\right)$, then we have the result.

Lemma 1.13。 Let $(f, s),(g, t)$ be pointed spaces over $B$. If $f: T(f) \rightarrow B$ is a Serre fibering whose fiber $F$ is path connected, and if ${ }_{B} H_{i}(f, s)=0$ for $i<k$, then ${ }_{B} H_{i}((g, t) \wedge(f, s))=0$ for $i<k$.

Proof. Since $s B$ is a retract of $T(f)$, the long exact sequence associated with the cofibering $s B \rightarrow T(f) \rightarrow T(f) / s B$ splits into short exact sequences $0 \rightarrow$ $H_{i}(s B) \rightarrow H_{i}(T(f)) \rightarrow \tilde{H}_{i}(T(f) / s B) \rightarrow 0 \quad(i=0,1,2, \ldots)$. The assumption implies that $H_{i}(s B) \rightarrow H_{i}(T(f))$ is an isomorphism for $i<k$. Hence $f_{*}: H_{i}(T(f)) \rightarrow H_{i}(B)$ is an isomorphism for $i<k$. Since the composition $H_{i}(F) \rightarrow H_{i}(T(f)) \rightarrow H_{i}(B)$ is zero unless $i=0$, we have $H_{i}(F)=0$ for $0<i<k$. Consider the Serre spectral sequence associated with the induced fibering $F \rightarrow T(g) \underset{B}{\mathbb{B}} T(f) \rightarrow T(g)$ by the map $g$. Then $E_{p, q}^{2}=H_{p}\left(T(g) ; H_{q}(F)\right)=0$ for $0<q<k$ implies $E_{p, q}^{\infty}=0$ for $0<q<k$. Hence we have $F_{i-1,1}=0$ for $i<k$ which yields that the edge homomorphism $H_{i}(T(g) \underset{B}{\times} T(f))=F_{i, 0} \rightarrow E_{i, 0}^{\infty} \subset E_{i, 0}^{2}=H_{i}(T(g))$ is injective for $i<k$. Since $T(g)$ is a retract of $T(g) \underset{B}{\times} T(f)$, it follows that $\bar{t}_{*}: H_{i}(T(g)) \rightarrow$ $H_{i}(T(g) \times \underset{B}{T} T(f))$ is an isomorphism for $i<k$. Noting that $\bar{t} T(g) \cap \bar{s} T(f)=\{*\}$ $\times s B$, consider the long exact sequence associated with the cofibering $T(g) \stackrel{t}{\longrightarrow}$ $\bar{t} T(g) \cup \bar{s} T(f) \rightarrow T(f) / s B$. Since $T(g)$ is a retract of $\bar{t} T(g) \cup \bar{s} T(f)$ and $\widetilde{H}_{i}(T(f) /$ $s B)=0$ for $i<k, \bar{t}: H_{i}(T(g)) \rightarrow H_{i}(\bar{t} T(g) \cup \bar{s} T(f))$ is an isomorphism for $i<k$. By the commutativity of the diagram

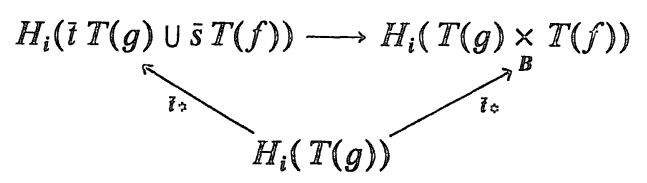

$H_{i}(\bar{t} T(g) \cup \bar{s} T(f)) \rightarrow H_{i}(T(g) \underset{B}{\times} T(f))$ is an isomorphism for $i<k$. Since $\Phi\left((g, t) \wedge_{B}(f, s)\right)$ is the cofiber of the inclusion $\bar{t} T(g) \cup \bar{s} T(f) \subset \mathbb{T}(g) \underset{B}{\times} T(f)$ by (1.10), we have ${ }_{B} H_{i}((g, t) \underset{B}{\wedge}(f, s))=0$ for $i<k$.

Lemma 1.14. Let $(f, s)$ be a pointed space over $B$.

(i) If $f: T(f) \rightarrow B$ is a Serre fibration, so is $C_{\varphi}(f): T\left(C_{\varphi}(f, s)\right) \rightarrow B$.

(ii) If the total space $T(f)$ is path connected, each fiber of $C_{\varphi}(f)$ : 
$T\left(C_{\varphi}(f, s)\right) \rightarrow B$ is also path connected.

(iii) If $B$ is path connected, so is the total space $T\left(C_{\varphi}(f, s)\right)$.

Proofs are straightforward from the construction of $C_{\varphi}$.

Corollary 1.15. Let $\tilde{h}_{*}$ be a connective homology theory and let $B$ be a simply connected space. Then, for pointed spaces $(g, t),(f, s)$ over $B$ such that $f: T(f) \rightarrow B$ is a Serre fibration, we have ${ }_{B} h_{q}\left((g, t){ }_{B}^{\wedge} C_{\varphi}^{p}(f, s)\right)=0$ for $q<2 p$ and $p \geqq 2$.

Proof. By the above lemmas, $C_{\varphi}^{p}(f): T\left(C_{\varphi}^{p}(f, s)\right) \rightarrow B$ is a Serre fibration whose fiber is path connected if $p \geqq 2$ and ${ }_{B} H_{q}\left(C_{\varphi}^{p}(f, s)\right)=0$ for $q<2 p$. Hence (1.12) implies ${ }_{B} H_{q}\left((g, t){ }_{B} C_{\varphi}^{p}(f, s)\right)=0$ for $q<2 p$ and $p \geqq 2$. Applying the Atiyah-Hirzebruch spectral sequence, the result follows.

It follows from (1.15) that $A_{p, q}=0$ for $p \leqq 0, q \in \mathbb{Z}$, and $\cap_{p \leqq 0} F_{p, n-p}=0$ (in fact, $F_{p, n-p}=0$ for $\left.p<\min \{-n,-1\}\right)$. Now we have a sufficient condition for the convergence.

Theorem 1.16. Assume that $\tilde{h}_{*}$ is a connective homology theory and $(f, s)$ is a pointed space over a simply connected space $B$ such that $f: T(f) \rightarrow B$ is a Serre fibration. Then, for any pointed space $(g, t)$ over $B$, the Künneth spectral sequence constructed in (1.7) converges to ${ }_{B} h_{*}\left((g, t){ }_{B}(f, s)\right)$.

In order to make an identification of the $E^{2}$-term in terms of homological algebra, we have to assume some conditions. Let $(f, s),(g, t)$ be pointed spaces over $B$.

Assumptions 1.17. (i) $\tilde{h}_{*}$ is a multiplicative homology theory on Top* (not necessarily connective).

(ii) $\tilde{h}_{*}\left(B_{+}\right)$is a flat $\tilde{h}_{*}\left(S^{0}\right)$-module.

(iii) Either $\tilde{h}_{*}(T(f) / s B)$ or $\tilde{h}_{*}(T(g) / t B)$ is flat over $\tilde{h}_{*}\left(S^{0}\right)$.

Under the above assumptions, the smash products $\tilde{h}_{*}\left(B_{+}\right) \otimes \tilde{h}_{*}(X) \rightarrow \tilde{h}_{*}\left(\left(B_{+}\right)\right.$ $\wedge X), \tilde{h}_{*}(X) \otimes \tilde{h}_{*}\left(B_{+}\right) \rightarrow \tilde{h}_{*}\left(X \wedge\left(B_{+}\right)\right)$are isomorphisms for any pointed space $\left(X, x_{0}\right)$, where the tensor products are taken over $\tilde{h}_{*}\left(S^{0}\right)$. Define $\psi^{L}: T(f) / s B$ $\rightarrow\left(B_{+}\right) \wedge T(f) / s B$ and $\psi^{R}: T(g) / t B \rightarrow T(g) / t B \wedge\left(B_{+}\right)$by $\psi^{L_{\circ}} \pi(x)=f(x) \wedge \pi(x)$ and $\psi^{R_{\circ}} \rho(y)=\rho(y) \wedge g(x)$ respectively, where $\pi: T(f) \rightarrow T(f) / s B$ and $\rho: T(g)$ $\rightarrow T(g) / t B$ are collapsing maps. Note that $\psi^{L}=\psi^{R}=($ the diagonal map of $B$ ) if $(f, s)=(g, t)=\Gamma\left(S^{0}, *\right)$. We put $C=\tilde{h}_{*}\left(B_{+}\right)\left(={ }_{B} \tilde{h}_{*}\left(\Gamma\left(S^{0}, *\right)\right)\right)$ and define a 
coproduct $C \rightarrow C \otimes C$ to be the composite of the map induced by the diagonal map and the inverse of the smash product. The counit $C \rightarrow \tilde{h}_{*}\left(S^{0}\right)$ is the map induced by the collapsing map $\varepsilon: B_{+} \rightarrow S^{0}$. Let us define a left coaction of $C$ on ${ }_{B} h_{*}(f, s)$ and a right coaction on ${ }_{B} h_{*}(g, t)$ as follows.

$$
\begin{aligned}
&{ }_{B} h_{*}(f, s)=\tilde{h}_{*}(T(f) / s B) \stackrel{\psi_{*}^{L}}{\longrightarrow} \tilde{h}_{*}\left(\left(B_{+}\right) \wedge T(f) / s B\right) \stackrel{\wedge^{-1}}{\cong} \\
& \tilde{h}_{*}\left(B_{+}\right) \otimes \tilde{h}_{*}(T(f) / s B)=C \otimes_{B} h_{*}(f, s) \\
&{ }_{B} h_{*}(g, t)=\tilde{h}_{*}(T(g) / t B) \stackrel{\psi_{*}^{R}}{\longrightarrow} \tilde{h}_{*}\left(T(g) / t B \wedge\left(B_{+}\right)\right) \stackrel{\wedge^{-1}}{\cong} \\
& \tilde{h}_{*}(T(g) / t B) \otimes \tilde{h}_{*}\left(B_{+}\right)={ }_{B} h_{*}(g, t) \otimes C
\end{aligned}
$$

Note that the following diagrams are commutative, where $T$ is the swiching map.

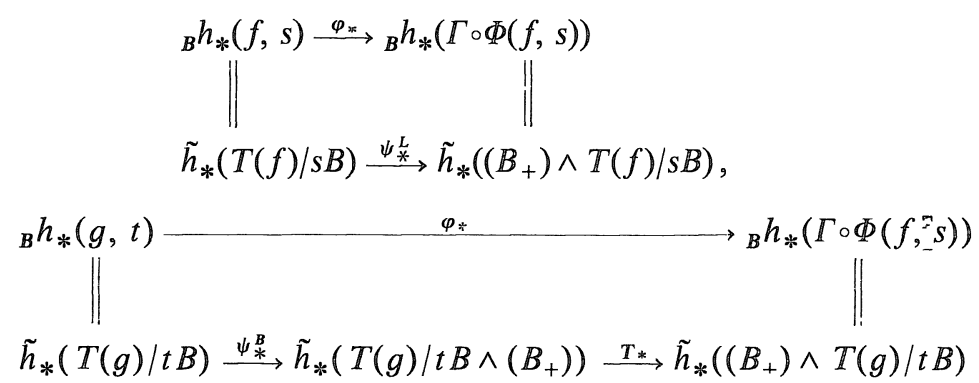

We construct a natural map $\theta:{ }_{B} h_{*}((g, t) \underset{B}{\wedge}(f, s)) \rightarrow_{B} h_{*}(g, t) \square_{B} h_{*}(f, s)$ under the assumption 1.17, where the cotensor products are taken over the coalgebra $C$. Let $\bar{s}, \bar{t}$ be maps defined in (1.10), then the composition of maps $T(g) \times T(f) / \bar{t} T(g) \cup \bar{s} T(f) \stackrel{\mu}{\longrightarrow} T(g) \times T(f) / T(g) \times s B \cup t B \times T(f)=T(g) /$ $t B \wedge T(f) / s B \stackrel{B \wedge \psi L}{\longrightarrow} T(s) / t B \wedge\left(B_{+}\right) \wedge T(f) / s B$ coincides with the composition $T(g)$ $\times T(f) / \bar{t} T(g) \cup \bar{s} T(f) \stackrel{\mu}{\longrightarrow} T(g) \times T(f) / T(g) \times s B \cup t B \times T(f)=T(g) / t B \wedge T(f) / s B$ $\stackrel{\underset{\psi R}{B} \wedge 1}{\longrightarrow} T(g) / t B \wedge\left(B_{+}\right) \wedge T(f) / s B$, where $\mu$ is induced by the inclusion $T(g) \times \underset{B}{ } T(f)$ $\subset T(g) \times T(f)$. Noting that the smash products $\tilde{h}_{*}(T(g) / t B) \otimes \tilde{h}_{*}(T(f) / s B)$ $\wedge \tilde{h}_{*}(T(g) / t B \wedge T(f) / s B)$ and $\tilde{h}_{*}(T(g) / t B) \otimes \tilde{h}_{*}\left(B_{+}\right) \otimes \tilde{h}_{*}(T(f) / s B) \wedge \tilde{h}_{*}(T(g) / t B$ $\left.\wedge\left(B_{+}\right) \wedge T(f) / s B\right)$ are isomorphisms, we see that the map ${ }_{B} h_{*}((g, t) \wedge(f, s))=$ $\tilde{h}_{*}(T(g) \times T(f) / \bar{t} T(g) \cup \bar{s} T(f)) \stackrel{\mu_{*}}{\longrightarrow} \tilde{h}_{*}(T(g) / t B \wedge T(f) / s B) \stackrel{\wedge^{-1}}{\longrightarrow} \tilde{h}_{*}(T(g) / t B) \otimes \tilde{h}_{*} \cdot$ $(T(f) / s B)={ }_{B} h_{*}(g, t) \otimes_{B} h_{*}(f, s)$ is lifted to the map $\theta$.

Lemma $\mathbb{1} 1 \mathbb{1}_{\circ}{ }_{B} \tilde{h}_{*}(\Gamma \circ \Phi(f, s))$ is an injective C-comodule. In fact the smash product gives an isomorphism as comodules

$C \otimes_{B} h_{*}(f, s)=\tilde{h}_{*}\left(B_{+}\right) \otimes \tilde{h}(T(f) / s B) \wedge \tilde{h}_{*}\left(\left(B_{+}\right) \wedge T(f) / s B\right)={ }_{B} h_{*}(\Gamma \circ \Phi(f, s))$.

Proof is straightforward. 
Lemma 1.19. The natural map $\theta:{ }_{B} h_{*}\left((g, t) \wedge{ }_{B} \Gamma \circ \Phi(f, s)\right) \rightarrow{ }_{B} h_{*}(g, t)$ $\square_{B} h_{*}(\Gamma \circ \Phi(f, s))$ is an isomorphism.

Proof. Just note that the geometric fact $T(g) \underset{B}{\times}(B \times T(f) / s B) \cong T(g) \times$ $T(f) / s B$ corresponds to the algebraic fact

$$
{ }_{B} h_{*}(g, t) \square\left(C \otimes_{B} h_{*}(f, s)\right) \cong{ }_{B} h_{*}(g, t) \otimes_{B} h_{*}(f, s) .
$$

Lemma 1.20。 $0 \rightarrow{ }_{B} h_{*}(f, s) \stackrel{\varphi_{*}}{\longrightarrow} h_{*}(\Gamma \circ \Phi(f, s)) \stackrel{\varphi_{* \circ l_{*}}}{\longrightarrow} h_{*}\left(\Gamma \circ \Phi \circ C_{\varphi}(f, s)\right) \rightarrow \cdots \rightarrow$ ${ }_{B} h_{*}\left(\Gamma \circ \Phi \circ C_{\varphi}^{i}(f, s)\right) \stackrel{\omega_{* \circ} \iota_{*}}{\longrightarrow}{ }_{B} h_{*}\left(\Gamma \circ \Phi \circ C_{\varphi}^{i+1}(f, s)\right) \rightarrow \cdots$ is an injective resolution of ${ }_{B} h_{*}(f, s)$.

Proof. The composition ${ }_{B} h_{*}\left(C_{\varphi}^{i}(f, s)\right) \stackrel{\varphi_{*}}{\longrightarrow} h_{*}\left(\Gamma \circ \Phi \circ C_{\varphi}^{i}(f, s)\right) \cong C \otimes_{B} h_{*}\left(C_{\varphi}^{i}(f\right.$, $s)) \stackrel{\varepsilon * \otimes 1}{\longrightarrow}{ }_{B} h_{*}\left(C_{\varphi}^{i}(f, s)\right)$ is the identity. Thus $\varphi_{*}$ is a monomorphism, and the long exact sequence associated with the cofibration $C_{\varphi}^{i}(f, s) \rightarrow \Gamma \circ \Phi \circ C_{\varphi}^{i}(f, s)$ $\rightarrow C_{\varphi}^{i+1}(f, s)$ splits into a short exact sequence $0 \rightarrow_{B} h_{*}\left(C_{\varphi}^{i}(f, s)\right) \stackrel{\varphi_{*}}{\longrightarrow}{ }_{B} h_{*}\left(\Gamma \circ \Phi_{\circ}\right.$ $\left.C_{\varphi}^{i}(f, s)\right) \stackrel{\iota_{*}}{\longrightarrow} h_{*}\left(C_{\varphi}^{i+1}(f, s)\right) \rightarrow 0$ of $C$-comodules. Splice these exact sequences for $i=0,1,2, \ldots$, and use (1.18), we have the result.

Remark 1.21. Under (i), (ii) of (1.17), the category of $C$-comodules becomes a relative abelian category $([1],[5])$ and ${ }_{B} h_{*}$ is a homology theory Top $_{*} / B \rightarrow$ (the category of $C$-comodules). By the proof of (1.20), the $C$-comodule homomorphism $\varphi_{*}:{ }_{B} h_{*}(f, s) \rightarrow{ }_{B} h_{*}(\Gamma \circ \Phi(f, s))$ is a split monomorphism as a $h_{*}\left(S^{0}\right)$-module homomorphism.

Theorem 1.22. Under Assumptions 1.17, the $E^{2}$-term of the Künneth spectral sequence is naturally isomorphic to $\operatorname{Cotor}_{*, *}^{C}\left({ }_{B} h_{*}(g, t),{ }_{B} h_{*}(f, s)\right)$ $\left(E_{p, q}^{2} \cong \operatorname{Cotor}_{p, q}^{C}\left({ }_{B} h_{*}(g, t),{ }_{B} h_{*}(f, s)\right)\right)$.

Proof. This follows from (1.19) and (1.20).

Remarks 1.23. (i) The edge homomorphism ${ }_{B} h_{*}((g, t) \wedge(f, s)) \rightarrow E_{0, n}^{2} \cong$ $\operatorname{Cotor}_{0, n}^{C}\left({ }_{B} h_{*}(g, t),{ }_{B} h_{*}(f, s)\right)={ }_{B} h_{*}(g, t) \square_{B} h_{*}(f, s)$ coincides with the natural $\operatorname{map} \theta$.

(ii) (1.17) is always satisfied if $\tilde{h}_{*}$ is ordinary homology theory over a field or Morava K-theory.

(iii) We consider the category of spaces over $B$, denoted by $\mathbb{T o p} / B$. An object of Top/B is a continuous map $f: T(f) \rightarrow B$, and a morphism $\varphi: f \rightarrow g$ in $\operatorname{Top} / B$ is a continuous map $T(\varphi): T(f) \rightarrow T(g)$ such that $g \circ T(\varphi)=f$. Define a functor $G: \mathbb{T o p} / B \rightarrow \mathbb{T o P} * / B$ as follows. Put $G(f)=\left(f_{+}, s_{f}\right)$ and $T(G(f))=T\left(f_{+}\right)$ 
$=T(f) \Perp B$ (disjoint union), $f_{+}$and $s_{f}$ are given by $f_{+}(x)=f(x)$ for $x \in T(f)$ $f_{+}(b)=b$ for $b \in B$ and $s_{f}(b)=b$. Then it is easy to verify that $\Phi(G(f))=T(f)_{+}$ and $\Phi\left(G(g) \wedge_{B} G(f)\right)=\left(T(g) \times{ }_{B} T(f)\right)_{+}$where $f$ and $g$ are spaces over $B$. Therefore, if a fiber product of $f$ and $g$ is given, the Künneth spectral sequence associated with $G(f)$ and $G(g)$ is the Eilenberg-Moore spectral sequence ([7], [8]).

\section{§2. A Relation betweer the Algebraic Bocksteim Homomorphism amd the Geometric Bockstein Homomorphism}

Throughout this section, we assume that $B$ is a simply connected space such that $H_{*}\left(B: \mathbb{Z}_{(l)}\right)$ is flat and that $(f, s),(g, t)$ are pointed spaces over $B$ such that both $H_{*}\left(T(f) / s B: \mathbb{Z}_{(l)}\right)$ and $H_{*}\left(T(g) / t B: \mathbb{Z}_{(l)}\right)$ are flat and $f: T(f) \rightarrow B$ is a Serre fibration, where $l$ is a fixed prime number. Note that a $\mathbb{Z}_{(l)}$-module is flat if and only if it is torsion free.

Lemma $\mathbb{2}_{0} \mathbb{H}_{0}$ Under the above assumptions, ${ }_{B} H_{*}\left(C_{\varphi}^{i}(f, s): \mathbb{Z}_{(l)}\right)(i=0,1$, $2, \ldots)$ is flat.

Proof. Inductively, assume that ${ }_{B} H_{*}\left(C_{\varphi}^{i}(f, s): \mathbb{Z}_{(l)}\right)$ is flat. The cofibration $C_{\varphi}^{i}(f, s) \stackrel{\varphi}{\longrightarrow} \Gamma \circ \Phi_{\circ} C_{\varphi}^{i}(f, s) \stackrel{\iota}{\longrightarrow} C_{\varphi}^{i+1}(f, s)$ gives a short exact sequence $0 \rightarrow_{B} H_{*}$ $\left(C_{\varphi}^{i}(f, s): \mathbb{Z}_{(l)}\right) \rightarrow_{B} H_{*}\left(\Gamma \circ \Phi \circ C_{\varphi}^{i}(f, s): \mathbb{Z}_{(l)}\right) \rightarrow_{B} H_{*}\left(C_{\varphi}^{i+1}(f, s): \mathbb{Z}_{(l)}\right) \rightarrow 0$ which splits as $\mathbb{Z}_{(l)}$-modules. By $(1.18),{ }_{B} H_{*}\left(\Gamma \circ \Phi \circ C_{\varphi}^{i}(f, s): \mathbb{Z}_{(l)}\right)$ is isomorphic to $H_{*}\left(B: \mathbb{Z}_{(l)}\right)$ $\otimes_{B} H_{*}\left(C_{\varphi}^{i}(f, s): \mathbb{Z}_{(l)}\right)$ which is also flat. Hence ${ }_{B} H_{*}\left(C_{\varphi}^{i+1}(f, s): \mathbb{Z}_{(l)}\right)$ is flat.

Consider two Künneth spectral sequences converging to ${ }_{B} H_{*}((g, t) \underset{B}{\wedge}(f, s)$ : $\left.\mathbb{F}_{l}\right)$ and ${ }_{B} H_{*}\left((g, t) \underset{B}{\wedge}(f, s): \mathbb{Z}_{(l)}\right)$. We put

$$
\begin{array}{ll}
D_{p, q}^{1}={ }_{B} H_{q}\left((g, t) \underset{B}{\wedge} C_{\varphi}^{-p}(f, s): \mathbb{F}_{l}\right), & \mathbb{E}_{p, q}^{1}={ }_{B} H_{q}\left((g, t) \underset{B}{\wedge} \Gamma \circ \Phi \circ C_{\varphi}^{-p}(f, s): \mathbb{F}_{l}\right) \\
\bar{D}_{p, q}^{1}={ }_{B} H_{q}\left((g, t) \underset{B}{\wedge} C_{\varphi}^{-p}(f, s): \mathbb{Z}_{(l)}\right), & \bar{E}_{p, q}^{1}={ }_{B} H_{q}\left((g, t) \underset{B}{\wedge} \Gamma \circ \Phi \circ C_{\varphi}^{-p}(f, s): \mathbb{Z}_{(l)}\right) .
\end{array}
$$

By (1.19) and (2.1), $\bar{E}_{p, q}^{1}$ is torsion free. Hence the Bockstein exact sequence associated splits into short exact sequences $0 \rightarrow \bar{E}_{p, q}^{1} \stackrel{l \times}{\longrightarrow} \bar{E}_{p, q}^{1} \stackrel{\rho}{\longrightarrow} E_{p, q}^{1} \rightarrow 0$. Note that the multiplication by $l$ and the $\bmod l$ reduction $\rho$ induce maps of exact couples. Taking the homologies of complexes $\left\{\bar{E}_{*, *}^{1}, \bar{d}^{1}\right\}$ and $\left\{\mathbb{E}_{*, *}^{1}, d^{1}\right\}$, we have the algebraic Bockstein homomorphism $\tilde{\delta}: \mathbb{E}_{p, q}^{2} \rightarrow \bar{E}_{p-1, q}^{2}$ as the boundary homomorphism.

Theorem 2.2. If $x \in E_{p, q}^{2}$ is a permanent cycle, $\tilde{\delta} x \in \bar{E}_{p-1, q}^{2}$ is also a 
permanent cycle. Let $\bar{x} \in F_{p, q}$ be the element of ${ }_{B} H_{*}\left((g, t) \underset{B}{\wedge}(f, s) ; \mathbb{F}_{l}\right)$ corresponding to $x$, then $\delta \bar{x} \in \bar{F}_{p-1, q}=\operatorname{Im}\left\{\bar{D}_{p-1, q}^{1} \rightarrow \bar{D}_{0, p+q-1}^{1}\right\}$ and $\delta \bar{x}$ corresponds to the permanent cycle $-\tilde{\delta x}$, where $\delta:{ }_{B} H_{n}\left((g, t) \underset{B}{\wedge}(f, s) ; \mathbb{F}_{l}\right) \rightarrow{ }_{B} H_{n-1}((g, t) \wedge \underset{B}{(f}$, $\left.s) ; \mathbb{Z}_{(l)}\right)$ is the geometric Bockstein homomorphism.

Remark 2.3. In the case $x=0$ in the $E^{\infty}$-term, the above statement means that $\tilde{\delta} x=0$ in the $E^{\infty}$-term. If $x \neq 0$ and $\tilde{\delta} x=0$ in each $E^{\infty}$-terms, we assert that $\delta \bar{x} \in \bar{F}_{p-2, q+1}$.

The following lemma implies the above theorem.

Lemma 2.4. Let $X \stackrel{i}{\longrightarrow} Y \stackrel{j}{\longrightarrow} Z$ be a cofibration such that $\widetilde{H}_{*}\left(Y ; \mathbb{Z}_{(l)}\right)$ is torsion free. Suppose that a space $W$ and a map $k ; Z \rightarrow W$ such that $\tilde{H}_{*}(W$; $\left.\mathbb{Z}_{(l)}\right)$ is torsion free are given. Let $\partial, \partial^{\prime} ; \tilde{H}_{q}\left(X ; \mathbb{F}_{l}\right) \rightarrow \tilde{H}_{q}\left(W ; \mathbb{Z}_{(l)}\right) / \operatorname{Im} k_{*} \circ j_{*}$ be the maps defined as follows, for each $x \in \tilde{H}_{q}\left(X ; \mathbb{F}_{l}\right)$, take $y \in \tilde{H}_{q}\left(Y ; \mathbb{Z}_{(l)}\right)$ such that $\rho y=i_{*} x$. We can take $z \in \tilde{H}_{q}\left(Z ; \mathbb{Z}_{(l)}\right)$ such that $l z=j_{*} y$. Then $\partial x$ is defined to be the image of $k_{*} z$ by the projection $\pi: \widetilde{H}_{q}\left(W: \mathbb{Z}_{(l)}\right) \rightarrow \widetilde{H}_{q}\left(W: \mathbb{Z}_{(l)}\right) /$ $\operatorname{Im} k_{*} \circ j_{*}$. On the other hand, there exists $z^{\prime} \in \tilde{H}_{q}\left(Z ; \mathbb{Z}_{(l)}\right)$ such that $\Delta z^{\prime}=\delta x$, where $\delta: \tilde{H}_{q}\left(X ; \mathbb{F}_{l}\right) \rightarrow \tilde{H}_{q-1}\left(X: \mathbb{Z}_{(l)}\right)$ is the Bockstein homomorphism and $\Delta: \widetilde{H}_{q}\left(Z ; \mathbb{Z}_{(l)}\right) \rightarrow \widetilde{H}_{q-1}\left(X ; \mathbb{Z}_{(l)}\right)$ is the boundary homomorphism. $\partial^{\prime} x$ is defined to be $\pi \circ k_{*} z^{\prime}$. Then $\partial=-\partial^{\prime}$ holds.

Proof. It is easy to check that $\partial$ and $\partial^{\prime}$ are well-defined. We may assume $X \subset Y$ and replace $\tilde{H}_{q}\left(Z ; \mathbb{Z}_{(l)}\right)$ by $H_{q}\left(Y, X ; \mathbb{Z}_{(l)}\right)$. Let $S_{*}(X)$ and $S_{*}(Y)$ be the singular chain complexes of $X$ and $Y$ with $\mathbb{Z}_{(l)}$-coefficients. For $x \in \tilde{H}_{q}\left(X ; \mathbb{F}_{l}\right)$, we take a chain $\sigma \in S_{q}(X)$ such that $x$ is represented by the cycle $\rho_{\#} \sigma$ ( $\rho_{\#}$ is the mod $l$ reduction map). We put $d \sigma=l \alpha\left(\alpha \in S_{q-1}(X)\right)$, where $d$ is the differential of $S_{*}(X)$. Since $\widetilde{H}_{q}\left(Y ; \mathbb{Z}_{(l)}\right)$ is torsion free, we can take a cycle $\bar{\sigma} \in S_{q}(Y)$ such that $\rho_{\sharp} \bar{\sigma}$ is homologous to $i_{\sharp} \circ \rho_{\sharp} \sigma$. Therefore $\sigma-\bar{\sigma} \in l S_{q}(Y)+d\left(S_{q+1}(Y)\right)$, and we put $\sigma-\bar{\sigma}=l \beta+d \tau\left(\beta \in S_{q}(Y), \tau \in S_{q+1}(Y)\right)$. Since $\bar{\sigma}$ is a cycle, $\bar{\sigma}+d \tau$ is also a cycle homologous to $\bar{\sigma}$. So we may replace $\bar{\sigma}+d \tau$ by $\bar{\sigma}$ and we have $\sigma=\bar{\sigma}+l \beta$. It follows from $d \sigma=l \alpha$ that $d \beta=\alpha$. Since $j_{\sharp} \bar{\sigma}=-l \beta,-d \beta=-\alpha$ represents $\Delta z^{\prime} \in \widetilde{H}_{q-1}\left(X ; \mathbb{Z}_{(l)}\right)$. On the other hand, $\delta x \in \tilde{H}_{q-1}\left(X: \mathbb{Z}_{(l)}\right)$ is represented by $\alpha$. This completes the proof.

Proof of (2.2). We put $X=\Phi\left((g, t) \wedge_{B} C_{\varphi}^{-p}(f, s)\right), Y=\Phi\left((g, t) \wedge \underset{B}{\wedge} \circ \Phi \circ C_{\varphi}^{-p}(f\right.$, $s)), \quad Z=\Phi\left((g, t) \wedge{ }_{B} C_{\varphi}^{-p+1}(f, s)\right), \quad W=\Phi\left((g, t) \wedge{ }_{B} \Gamma \Phi_{\circ} C_{\varphi}^{-p+1}(f, s)\right)$. Suppose $x \in$ $E_{p, q}^{2}$ is a permanent cycle. Let $x^{\prime} \in E_{p, q}^{1}=\tilde{H}_{q}\left(Y ; \mathbb{F}_{l}\right)$ be a cycle which represents $x$. Since $x^{\prime}$ is also a permanent cycle, there exist $y \in D_{p, q}^{1}=\widetilde{H}_{q}\left(X ; \mathbb{F}_{l}\right)$ such that 
$(1 \wedge \varphi)_{*} y=x^{\prime}$. Then, it is easy to see that $\partial y=\tilde{\delta} x$ in $\mathbb{E}_{p-1, q^{*}}^{2} \quad \partial y$ is an element coming from $\bar{D}_{p-1, q}^{1}=H_{q}\left(Z: \mathbb{Z}_{(l)}\right)$ by the definition of $\partial$. Thus $\tilde{\delta} x$ is a permanent cycle. By the preceding lemma, $\delta \bar{x}$ belongs to $\bar{F}_{p-1, q}$ and corresponds to $-\tilde{\delta} x$.

\section{§3. Om the Homology Suspensions}

Let $B$ be a path connected topological space with a base point $*$ and let $P B$ be the space of paths in $B$ starting from $*$. And let us denote by $p: P B \rightarrow B$ the evaluation map at 1 , and also denote by $i: * \rightarrow B$ the inclusion. We fix these notations throughout this section. The following lemma is easily verified.

Lemma 3.1. (i) The total space of $\Gamma \circ \Phi \circ G(p)$ is given by $(B \times P B) \Perp \mathbb{B}$ and the projection $(B \times P B) \Perp B \rightarrow B$ maps both $(b, l) \in B \times \mathbb{P B}$ and $b \in B$ to $b$. The section $B \rightarrow(B \times P B) \Perp B$ maps $b$ to $b$. Moreover, $\varphi: G(p) \rightarrow \Gamma \circ \Phi \circ G(p)$ is given by $T(\varphi)(l)=(l(1), l)$ for $l \in P B, T(\varphi)(b)=b$ for $b \in B$.

(ii) The total space of $C_{\varphi} \circ G(p)$ is the quotient space of $(P B \times I) \Perp(B \times P B)$ by the equivalence relation generated by $(l, 1) \sim(l(1), l)$ and $\left(l^{\prime}, 0\right) \sim\left(l^{\prime \prime}, 0\right)$ if $l^{\prime}(1)=l^{\prime \prime}(1)$. The projection is given by $[l, r] \rightarrow l(1),[b, l] \rightarrow b$ for $l \in P B, r \in \mathbb{I}$, $b \in B$, and the section is given by $b \rightarrow\left[l_{b}, 0\right]$, where $l_{b}$ is any element of $\mathbb{P B}$ such that $l_{b}(1)=b$. And the inclusion $\iota: \Gamma \circ \Phi \circ G(p) \rightarrow C_{\varphi} \circ G(p)$ is given by $T(c)$. $(b, l)=[b, l], T(c)(b)=\left[l_{b}, 0\right]$, where $b \in B, l \in P B$ and $l_{b}$ is as above.

We denote the total space of $C_{\varphi} \circ G(p)$ by $T_{B}$ and denote the projection and the section by $\tilde{p}: T_{B} \rightarrow B$ and $\tilde{s}: B \rightarrow T_{B}$.

Lemma 3.2. (i) Let $(f, s)$ be a pointed space over $B$, then we have $\Phi\left(G(i) \hat{B}_{B}(f, s)\right)=f^{-1}(*)$. In particular, we have $\Phi\left(G(i){ }_{B} G(p)\right)=\Omega B_{+}$, $\Phi\left(G(i) \wedge{ }_{B} \Gamma \circ \Phi \circ G(p)\right)=P B_{+}, \Phi\left(G(i) \wedge_{B} C_{\varphi} \circ G(p)\right)=P B \cup C \Omega B$, where $C \Omega B$ is the unreduced cone $\Omega B \times I / \Omega B \times\{0\}$ with a base point $\Omega B \times\{0\} / \Omega B \times\{0\}$ and we identify $\omega \in \Omega B(\subset P B)$ with $[\omega, 1] \in C \Omega B$. Moreover, $\Phi(1 \wedge \varphi)$ and $\Phi(1 \wedge \iota)$ are natural inclusions $\Omega B_{+} \rightarrow P B_{+}, P B_{+} \rightarrow P B \cup C \Omega B$.

(ii) $\Phi\left(G(i) \wedge \Gamma \circ \Phi \circ C_{\varphi} \circ G(p)\right)=\Phi \circ C_{\varphi} \circ G(p)=\mathbb{T}_{B} / \tilde{s} B=((P B \times I) \Perp$ $(B \times P B) /\left(\begin{array}{l}(l, 1) \sim(l(1), l) \\ \left(l^{\prime}, 0\right) \sim\left(l^{\prime \prime}, 0\right)\end{array}\right.$, and $\Phi(1 \wedge \varphi): P B \cup C \Omega B \rightarrow T_{B} / \tilde{s} B$ is given by $\Phi(1 \wedge \varphi)$. $([l])=[*, l], \Phi(1 \wedge \varphi)([\omega, t])=[\omega, t]$

Proof. (i) is straightforward. (ii) is verified by applying (1.10), (iii).

Lemma 3.3. Define $\pi: T_{B} / \tilde{s} B \rightarrow B$ by $\pi[b, l]=b, \pi[l, t]=l(t)$, then $\pi$ is $a$ 
natural (stable) homotopy equivalence and the diagram

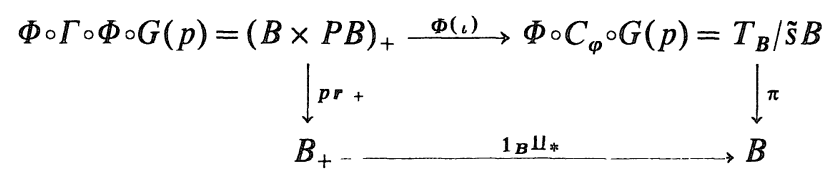

is commutative, where $p r: B \times P B \rightarrow B$ is the projection $\operatorname{pr}(b, l)=b$.

Proof. Commutativity of the above diagram is obvious. Consider the following homotopy commutative diagram.

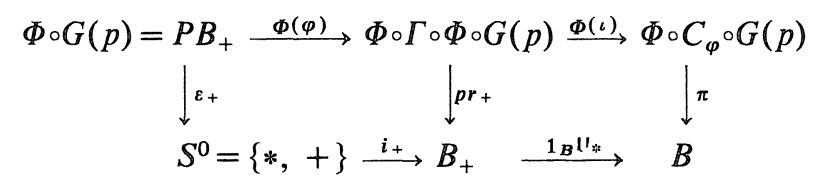

where $\varepsilon: P B \rightarrow *$. The both horizontal rows are cofiber sequences and $\varepsilon_{+}$and $p r_{+}$are homotopy equivalences. Hence we have the result.

Lemma 3.4. Let $c: P B \cup C \Omega B \rightarrow \sum \Omega B$ be the map which collapses $P B \cup$ $\left\{\left[\omega_{0}, r\right] \mid \omega_{0}\right.$ is the constant loop at $\left.*\right\}$ to the base point and let $\sigma: \sum \Omega B \rightarrow B$ be the adjoint of the identity map of $\Omega B$; that is, $\sigma$ is defined by $\sigma([\omega, t])=\omega(t)$. Then, $c$ is a homotopy equivalence and the following diagram is commutative.

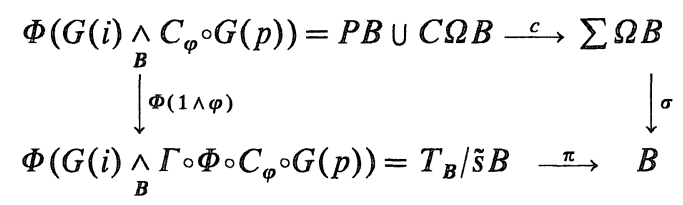

Proof. It is obvious that $c$ is a homotopy equivalence, and we can verify that the diagram commutes, applying (3.2) and (3.3).

Let $\tilde{h}_{*}$ be a multiplicative homology theory on $\operatorname{Top}_{*}$, and let us consider the Künneth spectral sequence associated with $\tilde{h}_{*}$ and the pointed spaces $G(i)$, $G(p)$. In other words we consider the Eilenberg-Moore spectral sequence associated with the path fibering $\Omega B \rightarrow P B \rightarrow B$. The preceding lemma implies the following.

Theorem 3.5. Assume that $h_{*}(B)$ is a flat $h_{*}(p t)$-module. Then $F_{-1, n+1}$ $=\tilde{h}_{n}(\Omega B)=\operatorname{ker}\left\{h_{n}(\Omega B) \rightarrow h_{n}(p t)\right\}$ and the composition $\tilde{h}_{n}(\Omega B)=F_{-1, n+1} \rightarrow F_{-1, n+1}$ $\mid F_{-2, n+2} \subset E_{-1, n+1}^{\infty} \subset E_{-1, n+1}^{2} \cong P h_{n+1}(B) \subset h_{n+1}(B)$ coincides with the homology suspension $\tilde{h}_{n}(\Omega B) \cong \tilde{h}_{n+1}\left(\sum \Omega B\right) \stackrel{\sigma m}{\longrightarrow} h_{n+1}(B)$, where $P h_{*}(B)$ is the submodule of $h_{*}(B)$ consists of primitive elements which is naturally identified with $\operatorname{Cotor}_{-1, *}^{h_{*}(B)}$. 
$\left(h_{*}(p t), h_{*}(p t)\right)$ and we identify $E_{-1, n+1}^{2}$ with $\operatorname{Cotor}_{-1, n+1}^{h_{-1}(B)}\left(h_{*}(p t), h_{*}(p t)\right)$ as in Section 1.

Corollary 3.6. Under the same assumption as above, if the homology suspension $\sigma_{*}: \tilde{h}_{n}(\Omega B) \rightarrow h_{n+1}(B)$ maps surjectively onto $P h_{n+1}(B)$, every element of $E_{-1, n+1}^{2}$ is a permanent cycle. Assume further that the Künneth spectral sequence converges. If every element of $\mathbb{E}_{-1, n+1}^{1}$ is a permanent cycle, $\sigma_{*}$ maps $\tilde{h}_{n}(\Omega B)$ surjectively onto $P h_{n+1}(B)$.

\section{References}

[1] Eilenberg, S. and Moore, J. C., Foundations of relative homological algebra, Memoirs A.M.S., 55 (1965).

[2] —, Homology and fibrations I, Comment. Math. Helv., 40 (1966), 199-236.

[ 3 ] Heller, A., Abstract homotopy in categories of fibrations and the spectral sequence of Eilenberg and Moore, Illinois J. Math., 16 (1972), 454-474.

[4] Hodgkin, L. H., The equivariant Künneth theorem in $\mathbb{K}$-theory, Lecture Notes in Math., No. 496, Springer-Verlag, Berlin-Heiderberg-New York, 1975.

[5] MacLane, S. S., Homology, Band $\mathbb{1} \mathbb{1 4}$, Springer-Verlag, Berlin-Heiderberg-New York, 1963.

[6] Rector, D. L., Steenrod operations in the Eilenberg-Moore spectral sequence, Comment, Math. Helv., 45 (1970), 540-552.

[ 7 ] Smith, L., Lectures on the Eilenberg-Moore spectral sequence, Lecture Notes in Math., No. 134 , Springer-Verlag, Berlin-Heiderberg-New York, 1970.

[ 8 ] Smith, L., On the Künneth theorem I, Math. Zeit., 116 (1970), 94-140.

[9] Switzer, R. M., Algebraic Topology-Homotopy and Homology, Band 212, SpringerVerlag, Berlin, 1975.

[10] Yamaguchi, A., The homology of double loop spaces of complex Stiefel manifolds, to appear.

[11] Johnson, D. C., Miller, H. R., Wilson, W. S. and Zahler, R. S., Boundary homomorphism in the generalized Adams spectral sequence and the nontriviality of infinitely many $\gamma_{t}$ in the stable homotopy, Notas de matematicas y Simposia, Numero $\mathbb{1}$; Reunion Sobre Teoria de Homotopia, ed. D. Davis, 29-46. Sociedad Matematica Mexicana, Mexico, D.F., 1975. 
